\title{
A competitividade internacional: notas para uma abordagem não ortodoxa
}

\author{
The international competitiveness: \\ notes for a non-orthodox approach
}

ENÉAS GONÇALVES DE CARVALHO*

SEBASTIÃO NETO RIBEIRO GUEDES**

RESUMO: O objetivo deste pequeno artigo é contribuir para a abordagem não ortodoxa da Competitividade Internacional (C. I.). A perspectiva teórica adotada é a neoschumpeteriana combinada com o enfoque das chamadas “capacidades dinâmicas". Do ponto de vista metodológico, parece apropriado qualificar este trabalho como histórico-comparativo.

PALAVRAS-CHAVE: Competitividade Internacional (C. I.); determinantes da C. I.; capacidade dinâmicas.

ABSTRACT: The purpose of this short article is to contribute to the unorthodox approach of the International Competitiveness (I. C.). The theoretical perspective adopted is the neoSchumpeterian combined with the so-called "dynamic capabilities" approach. From a methodological viewpoint, it seems appropriate to describe this work as a comparativehistorical one.

KEYWORDS: International Competitiveness (I. C.); determinants of I. C.; dynamic capabilities.

JEL Classification: D2; F19; F59; F69.

\section{INTRODUÇÃO}

A utilização da noção de competitividade parece ter sua origem na literatura das escolas de business (CIPR, 2013; Fagerberg, 2002). A chamada questão da competitividade ganhou destaque entre autores norte-americanos, a partir dos anos 1980, no âmbito da discussão sobre o fraco desempenho das empresas daquele país

\footnotetext{
* Professor assistente do Departamento de Economia da Universidade Estadual Paulista (Unesp), São Paulo/SP, Brasil. E-mail: egcarvalho58@gmail.com.

* Professor assistente do Departamento de Economia da Universidade Estadual Paulista (Unesp), São Paulo/SP, Brasil. E-mail: sebaneto@fclar.unesp.br. Submetido: 2/Agosto/2016; Aprovado: 31/Janeiro/2018.
} 
vis- $\grave{a}$-vis as suas concorrentes estrangeiras, especialmente as japonesas, em setores em que as empresas norte-americanas usualmente lideravam (Nelson, 1992; Morewy e Nelson, 1999).

Este tema perdeu grande parte do seu glamour (e parte da sua urgência), a partir do início dos anos 2000, em virtude do desempenho favorável da economia norte-americana na última década do século XX, contrastando com os decepcionantes desempenhos dos seus principais rivais: as economias japonesa (especialmente) e alemã (Berger, 2005, 2013).

Mais recentemente, entretanto, a questão da Competitividade Internacional parece ter recuperado parte do seu interesse anterior - especialmente nos países que têm enfrentado mais dificuldade na recuperação da grande crise de 2007-2008 e/ou já não vinham apresentando, desde antes da crise, um desempenho industrial muito satisfatório. Exemplos do interesse recente por este assunto e questões correlatas tanto no âmbito acadêmico quanto no campo governamental não são poucos ${ }^{1}$.

O objetivo deste pequeno artigo é contribuir para a abordagem não ortodoxa da Competitividade Internacional (C. I.). A perspectiva teórica adotada é a neoschumpeteriana combinada com o enfoque das chamadas 'capacidades dinâmicas'. Do ponto de vista metodológico, parece apropriado qualificar este trabalho como histórico-comparativo. Ele está organizado da seguinte maneira. A esta introdução se seguem mais quatro itens, onde são abordados, respectivamente, a noção de C. I., os determinantes da C. I. e a C. I. de um país. No último item são feitas as breves considerações finais.

\section{A NOÇÃO DE COMPETITIVIDADE INTERNACIONAL²}

Pode-se considerar a noção de competitividade, em uma primeira aproximação, como sendo o êxito relativo de uma unidade econômica - um agente individual (uma empresa), ou um grupo de agentes (um setor industrial), ou um agente coletivo $^{3}$ (um país, por exemplo) - no processo competitivo (no ambiente econômico)

\footnotetext{
${ }^{1}$ Do primeiro campo, apenas para citar os exemplos de maior destaque, pode-se mencionar: Making in America: from innovation to market (Berger, 2013); Rising above the gathering storm revisited: rapidly approaching category 5 (NAS, 2010); e Future of U.S. manufacturing - a literature review (partes I, II e III) (MIT, 2011, 2012). Do âmbito governamental, pode-se mencionar: The competitiveness and innovative capacity of the United States (DOC, 2012) e The European competitiveness report (EU, 20012). Este último é uma publicação bianual da União Europeia.

${ }^{2}$ Em função da restrição de espaço imposta a este artigo curto, não será possível tratar aqui, diretamente, do impacto e das mudanças que o avanço do processo de globalização tem produzido para a competitividade, seja das empresas, seja dos países. Assim sendo, informa-se ao leitor que neste e nos próximos dois itens está suposto na discussão o advento e o avanço do relevante processo, bem como as suas consequências não negligenciáveis.

${ }^{3}$ Um país pode ser considerado, a princípio, como uma mera agregação de agentes individuais (empresas) ou como um agente coletivo e, portanto, dotado de unidade.
} 
no qual ele se encontra inserido, num dado período de tempo. Analiticamente, o ambiente econômico dos agentes também pode ser considerado de forma mais ou menos abrangente - mercado/indústria, setor industrial, economia nacional, bloco regional ou economia internacional ${ }^{4}$ (OCDE, 1992; Possas, 1999; Carvalho, 2003).

Do ponto de vista teórico, partindo-se da perspectiva de que o mercado é o locus privilegiado da concorrência intercapitalista - isto é, que ele é o espaço, por excelência, do processo de interação das unidades econômicas (em sua busca incessante por lucros, através do esforço inovativo contínuo) -, parece adequado considerar o mercado como o espaço onde se desenvolve, principalmente, o processo competitivo e onde a competitividade de cada agente é, fundamentalmente, determinada (Schumpeter, 1984; Possas, 1985).

Tal perspectiva não implica nem considerar a competitividade como tendo lugar de forma isolada nem desconsiderar as possibilidades analíticas (ou mesmo teóricas) de enfocar a competitividade internacional de uma maneira mais abrangente - em termos do conjunto do setor industrial ou mesmo de um país (Morewy e Nelson, 1999; Nelson, 1992; OCDE, 1992).

A abordagem da competitividade enfatizando a dimensão setorial deste processo significa também admitir, por um lado, que as dimensões mais gerais da competitividade terão efeitos distintos nos diferentes mercados/indústrias ${ }^{5}$. Tal situação torna inviável, por outro lado, a possibilidade de uma simples extensão desta noção de competitividade, para âmbitos mais amplos, mediante uma mera agregação do mesmo. De fato, tal limitação se coloca porque a heterogeneidade intersetorial ultrapassa as distinções quanto à introdução de inovações (não apenas tecnológicas), mas compreende, também, diferentes potenciais de crescimento, de geração de renda etc. (Dosi et al., 1990; OCDE, 1992).

Tais diferenças qualitativas entre os diversos setores industriais resultam, em geral, em significativas diferenças dinâmicas, dado que os padrões de alocação das economias nacionais (ou mesmo regionais) não são iguais. Em outras palavras, o país que for mais competitivo nos setores que se caracterizam por maior elasticidade-renda da demanda, maior dinamismo tecnológico e maior crescimento no comércio internacional será mais competitivo do que os outros. Deste fato resulta que a agregação, neste caso, não é neutra e que, não é indiferente - também da perspectiva da C.I. de um país - a composição de setores em que o país é mais competitivo relativamente (Dosi et al., 1990; OCDE, 1992).

Uma definição de competitividade nacional compatível com as considerações

\footnotetext{
${ }^{4}$ Deve ser notado que, com o avanço dos processos de globalização produtiva, comercial e financeira nas últimas décadas (OCDE, 1992), parece cada vez mais apropriado considerar, a princípio, a competitividade em termos internacionais.

${ }^{5}$ Está sendo considerado aqui, como proposto por Possas (1985), que as noções de mercado (ou de estrutura de mercado) e de indústria podem ser entendidas como "praticamente" intercambiáveis. Além das razões teóricas e metodológicas apontadas pelo autor, existe ainda uma forte razão de natureza prática que reforça a mencionada escolha: o fato de que a disponibilidade de informações relativas ao âmbito industrial é bem maior do que a disponível para o âmbito de mercado.
} 
acima, e que se tornou, também, bastante influente entre os economistas não ortodoxos, foi adotada pela OCDE $(1992)^{6}$ (ver o penúltimo item, abaixo). A partir do mencionado estudo, pode se postular que a competitividade da economia de um país é mais do que a mera soma da competitividade coletiva ou "média" de suas empresas e está também vinculada aos seus padrões setoriais de produção e à sua inserção nos mercados internacionais respectivos (Dosi et al., 1990).

Independentemente do nível de abrangência adotado, a noção de competitividade tem que ser considerada em relação ao tempo - seja porque o ambiente econômico muda, seja porque as capacidades dos agentes podem se alterar através dos processos de aprendizado (o que também pode ter consequências para o ambiente econômico) -, dado que a competitividade é relativa e, também, mutável ao longo do tempo (Teece et al., 1997; Carvalho, 2003).

Ainda em relação à dimensão temporal, é possível tratar a competitividade de maneira mais ou menos abrangente. Tendo em vista, por um lado, a perspectiva schumpeteriana de que a introdução de inovações é a forma mais eficiente de concorrer - e, em consequência, uma arma fundamental para obter êxito relativo no processo competitivo -, e que, por outro, o processo de introdução de inovações é, habitualmente, de longa duração, parece mais apropriado considerar a noção de competitividade do ponto de vista do longo prazo (Schumpeter, 1984) ${ }^{7}$. Além disso, o longo prazo parece se revelar mais adequado para abordar, também, as dimensões dinâmicas (as mudanças) do processo competitivo, e, portanto, se qualifica como ponto de vista mais apropriado para o enfoque da competitividade (Carvalho, 2003; Possas, 1999).

Uma outra questão, também concernente à dimensão temporal, diz respeito às possibilidades de se considerar a competitividade de forma ex-ante ou ex-post (ou de ambas). Como em outros casos, também neste há questões teóricas e metodológicas implicados na eventual escolha. Com base no ponto de vista adotado aqui, a competitividade ex-post - corresponderia ao desempenho relativo do agente (ou agentes) econômico no processo competitivo, em um período passado de tempo. Analogamente, a competitividade ex-ante corresponderia ao desempenho relativo esperado (potencial) num intervalo de tempo futuro, mais ou menos próximo (CIPR, 2013; Carvalho, 2003; Possas, 1999).

Assim considerada, a noção de competitividade ex-ante implicaria excluir as eventuais alterações imprevistas no ambiente competitivo bem como o resultado da interação entre as estratégias adotadas pelos diversos agentes - que tampouco poderiam ser previstas com exatidão (Dosi, 2006). A diferença entre a competiti-

\footnotetext{
6 “Competitividade para uma nação é o grau com que ela pode, sob condições de mercado justo e livre, produzir bens e serviços que passem no teste dos mercados internacionais ao mesmo tempo em que mantém [ou] expande a renda real dos seus cidadãos. Competitividade é a base para um padrão de vida nacional. É também fundamental para a expansão das oportunidades de emprego e para permitir que a nação cumpra adequadamente com suas obrigações internacionais” (OCDE, 1992: 247).

${ }^{7}$ Existem também razões de ordem metodológica que reforçam a opção pelo longo prazo (ver adiante).
} 
vidade ex-ante e a ex-post resultaria, assim, da ocorrência de modificações no ambiente competitivo, e/ou nas capacidades das empresas e/ou das estratégias, nem sempre previsíveis, que venham a ser implementadas pelos concorrentes mais importantes $^{8}$ (Carvalho, 2003).

\section{DETERMINANTES DA COMPETITIVIDADE INTERNACIONAL}

Uma das maiores dificuldades ao se procurar abordar a noção de competitividade advém da sua complexidade intrínseca. A não ser para enfoques muito restritivos e heroicos, a competitividade decorre de um processo complexo e que tem vários determinantes. Além disso, a sua mensuração - a elaboração de indicadores de competitividade - tem se revelado um exercício que enfrenta inúmeras dificuldades ${ }^{9}$ (Dosi et al., 1990; OCDE, 1992; CIPR, 2013; Possas, 1999; Carvalho, 2003).

Com relação aos determinantes da competitividade, a abordagem das capacidades dinâmicas, proposta por Teece et al. (1997) e Teece (2007), parece conter os ingredientes mais adequados para explicar o êxito competitivo das empresas. Deste ponto de vista, a competitividade resultaria dos processos organizacionais e administrativos (ou "rotinas") adotados, das posições (ou ativos, no sentido amplo) obtidas e das trajetórias abertas às empresas - não se limitando apenas as trajetórias tecnológicos e, também, incluindo as estratégias implementadas (Teece et al., 1997; Tidd et al., 2005; Nelson, 2006).

O papel das estratégias, que nos trabalhos de Teece et al. (1997) e Teece (2007) não fica tão evidenciado, foi adequadamente esclarecido no livro de Tidd et al. (2005). Eles propuseram abordar as estratégias como estando entre os caminhos (trajetórias) que estão à disposição das empresas: "As estratégias empresariais que não reconhecem as complexidades do presente, bem como as incertezas associadas com a mudança e o futuro [...] estarão provavelmente erradas e serão potencialmente desastrosas se forem totalmente implementadas" (2005: 136).

Não parece ocioso lembrar que este enfoque baseado nas capacidades dinâmicas não é incompatível nem significa desprezar os comentários anteriores, quanto à circunstância de que a competitividade não é resultado apenas das interações no âmbito dos mercados/indústria. De fato, ela também é determinada por interações entre as empresas, as políticas econômicas (industriais, tecnológicas, comerciais, câmbio/monetária etc.) e as instituições (sistemas nacionais e/ou setoriais de ino-

\footnotetext{
${ }^{8}$ Talvez não seja ocioso lembrar que, na ausência de mudanças imprevistas ou na hipótese de que o futuro possa ser adequadamente previsto - pelo menos no que concerne às variáveis econômicas relevantes, aí incluídas as estratégias dos concorrentes mais importantes -, a distinção entre competitividade ex-ante e ex-post seria meramente formal e, portanto, desnecessária (Carvalho, 2003). Tal hipótese nos parece, entretanto, amplamente irrealista.

9 "Qualquer destes indicadores pode ser influenciado por outros fatores que não [pela] competitividade" (OCDE, 1992: 242).
} 
vação etc.) (Cantwell, 2005; Nelson, 1992, 2006; Morewy e Nelson, 1999; OCDE, 1992; CIPR, 2013).

\section{COMPETITIVIDADE INTERNACIONAL DE UM PAÍS}

Como tem sido apontado por vários autores, não se pode deixar de reconhecer que os países (e os seus governos) estão interessados não apenas na C. I. das suas empresas e, num sentido amplo, também se preocupam com a C. I. geral das suas economias e/ou do conjunto de suas indústrias. Em suma, os países - pelo menos os mais relevantes - não estão alheios à disputa competitiva entre eles, que, em algumas conjunturas, pode ser tornar até mesmo exacerbada (Gilplin, 2004; OCDE, 1992; Brenner, 2003; Bresser-Pereira, 2010).

Mesmo adotando-se a interpretação que o locus da C. I. é o mercado (como se faz neste artigo), permanece a questão de como enfocar a C. I. geral de uma economia e/ou de uma indústria como um todo - seja por uma razão prática e/ou analítica, seja por uma razão teórica/metodológica. “A ideia de uma diferença entre os níveis micro e macro refere-se à ideia de sistemas, e do que estes não são (porque são mais do que) a soma de suas partes componentes" (Dosi, 2006: 156). "Os sistemas certamente mudam por meio de alterações em suas partes, mas cada parte é definida contextualmente em relação ao sistema, e as direções da mudança do sistema podem ser diferentes das de cada parte específica" (Simon, 1981).

Ou seja, a não aceitação da interpretação reducionista, como explicitado acima, sugere, alternativamente, a adoção da abordagem sistêmica ${ }^{10}$ - na perspectiva da teoria da auto-organização ${ }^{11}$. Este enfoque parece encaminhar, também, à admissão da existência de propriedades emergentes ${ }^{12}$ - que parecem caracterizar os sistemas dinâmicos complexos abertos, especialmente os sociais (Edquist, 2006; Hodgson, 1997; Kauffman, 1993).

Deve-se notar, todavia, que a recusa ao reducionismo não implica adotar, como pensam alguns, certos tipos de perspectivas holísticas metafísicas ${ }^{13}$, que rejeitam a

\footnotetext{
10 "Um sistema consiste de dois tipos de constituintes: são eles, primeiro, alguns tipos de componentes e, segundo, relações entre eles [os componentes]. Os componentes e as relações devem formar um todo coerente (que tem propriedades diferentes das propriedades dos [seus] constituintes)" (Edquist, 2006: 187).

11 "A teoria da auto-organização trata de sistemas dinâmicos complexos abertos para o ambiente em termos da troca de matéria, energia e informação e composto de um número de subsistemas interagindo" (Silverberg, 1988: 531; Kauffman, 1993).

12 “ Emergência se refere à ideia que novas propriedades podem 'emergir' em um sistema complexo que não são redutíveis aos microelementos constituintes do 'menor nível'” (Hodgson, 1997: 156). Ver também Mayr (2008).

${ }^{13}$ Mayr (2008), por exemplo, sustenta, de maneira convincente, que nem todo holismo é metafísico (ou vitalista, no caso da biologia); defende a cientificidade de um certo holismo que, no caso da biologia, seria mais bem descrito pelo termo "organicismo".
} 
análise, e que conferem ao todo uma precedência relativamente aos seus elementos constituintes (Mayr, 2008).

Mas como abordar, então, uma noção correspondente de C. I. de um país e/ou do conjunto de sua indústria? Tentativas promissoras de enfrentar esta questão, não trivial, já foram realizadas por alguns autores. Nesta perspectiva, Dosi et al. (1990) propõem aceitar, por exemplo, a sugestão de Cohen et al. (1984) ${ }^{14}$. Para estes últimos, "[a] Competitividade Internacional no nível nacional é baseada no desempenho superior da produtividade e na habilidade econômica de deslocar a produção para atividades de produtividade mais elevadas, que por sua vez podem geral altos níveis de salário real" 15 (Cohen et al., 1984, apud Dosi et al.,1990: 150; Fagerbrg, 1988; OCDE, 1992).

A ideia subjacente a este tipo de abordagem é que, da perspectiva de um país, uma noção de C. I. tem que tratar, ao mesmo tempo, tanto das restrições externas, quanto da geração de bem-estar econômico (CIPR, 2013). Mesmo porque, com o decorrer do processo de globalização, a compatibilidade entre os interesses das empresas, dos governos e dos seus cidadãos (trabalhadores e consumidores) não têm, necessariamente, crescido (Berger, 2005; Bresser-Pereira, 2010).

Considere-se, a título de exemplo, o processo de segmentação da produção através das cadeias de valor. Os resultados (vantagens/desvantagens) da sua expansão para empresas, governos e cidadãos não são, necessariamente, convergentes. A intensificação deste processo, que tem ocorrido nas últimas décadas, pode ser muito funcional para as empresas, mas pode provocar, simultaneamente, problemas de balanço comercial e de geração de emprego para os países (Berger, 2005, 2013; Gilpin, 2004).

Da perspectiva dos seus cidadãos, os resultados podem ser contraditórios (pelo menos parcialmente). A redução de custos - possibilitada pela internacionalização do fornecimento de partes, peças e de bens finais e que pode ser parcialmente transferida aos preços - será favorável do ponto de vista dos consumidores. Por outro lado, o acréscimo das importações a ele associado - se não for acompanhado pela expansão compensatória das exportações - terá, entretanto, efeitos deletérios para a geração de emprego e de renda, além do impacto negativo no balaço comercial (Berger, 2005, 2013; Gilpin, 2004).

\footnotetext{
${ }^{14}$ No mesmo sentido, ver também Fagerberg (1988); OCDE (1992) e CIPR (2013).

15 “Ela [a C. I.] não é apenas uma medida da habilidade da nação de vender no exterior e manter o equilíbrio no comércio. Os países mais pobres do mundo são frequentemente capazes de fazer isto muito bem. Antes, ela é [a C.I.] a habilidade em competir internacionalmente naquelas commodities e serviços capazes de se constituírem em uma grande parte do consumo mundial e do valor adicionado no futuro"(Cohen et al., 1984, apud Dosi et al., 1990: 150). A propósito, ao abordar um tema relacionado, Bresser-Pereira (2010) destacou, apropriadamente, a importância da taxa de câmbio para manutenção da competitividade dos países de renda média que sofrem com a tendência à valorização das respectivas taxas.
} 


\section{CONSIDERAÇÕES FINAIS}

Estas últimas qualificações parecem reforçar a necessidade de abordar a C. I. não apenas no âmbito dos mercados, mas também da perspectiva dos países e/ou do conjunto de suas indústrias, uma vez que a simples transposição do enfoque micro para o plano macroeconômico/agregado parece enfrentar, também neste caso, dificuldades tanto analíticas quanto metodológicas/teóricas.

A existência destas duas dimensões distintas da C. I. parece ter sido reiterada nas últimas décadas. De fato, com o advento da globalização, a natureza e a intensidade do processo competitivo foram radicalmente alterados e a competitividade internacional tornou-se uma das principais preocupações das empresas e dos governos (OCDE, 1992, 1999; Berger, 2005, 2013; Bresser-Pereira, 2010).

Para encerrar, um esclarecimento. Também em razão da restrição de espaço, não foi possível abordar aqui, nem de forma introdutória, a intrincada (complicada) questão da mensuração da C. I. Como forma de minimizar esta lacuna, remete-se os leitores a dois trabalhos da bibliografia (Possas, 1999) e Carvalho (2003) onde se encontram análises equilibradas e bastante úteis deste problemático tópico metodológico.

\section{REFERÊNCIAS BIBLIOGRÁFICAS}

Berger, S. (2013) Making in America: from innovation to market. Cambridge: MIT Press.

Berger, S. (2005) How we compete. Nova York: Doubleday.

Brenner, R. (2003) O Boom e a bolha: os Estados Unidos na economia mundial. Rio de Janeiro: Record.

Bresser-Pereira, L. C. (2010) Globalização e competição: por que alguns países emergentes têm sucesso e outros não. São Paulo: Campus.

Cantwell, J. (2005) “Innovation and competitiveness". In: Fagerberg, J. et al., The Oxford handbook of Innovation. Oxford: Oxford Press.

Carvalho, E. G. de (2003) Globalização e estratégias competitivas na indústria automobilística: uma abordagem a partir da das principais montadoras instaladas no Brasil. Tese de Doutoramento. Unicamp.

CIPR (2013) Competitive Industrial Performance Report. Viena: UNIDO.

DOC (2012) The Competitiveness and innovative capacity of the United States Department of commerce. Washington.

Dosi, G. (2006) Mudança técnica e transformação industrial. Campinas: Unicamp.

Dosi, G., Pavitt, K. e Soete, L. (1990) Technical change and international trade. Hertfordshire: Harvester.

Edquist, C. (2006)"System of innovation: perspectives and challenges". In: Fagerberg, J. et al., The Oxford handbook of Innovation. Oxford: Oxford Press.

EU (2012)The European competitiveness report. European Union, 2012.

Fagerberg, J. (1988) “International competitiveness". The Economic Journal, 98: 355-74.

Fagerberg, J. (2002) Technology, grow and competitiveness. Cheltenham: E.E. publishing.

Gilpin, R. (2004) O Desafio do capitalismo global: a economia mundial no século XXI. Rio de Janeiro: Record.

Hodgson, G. M. (1997) Economia e evolução: o regresso da vida à teoria econômica. Oeiras: Celta. 
Kauffman, S. (1993) Origins of order: self-organization and selection in evolution. Oxford: Oxford Press.

MIT (2011e 2012) Future of U.S. manufacturing - a literature review (partes: I, II e III). Cambridge. Mayr, E.(2008) Isto é biologia: a ciência do mundo vivo. São Paulo: Companhia das Letras Mowery, D e Nelson, R. (1999) Sources of Industrial leadership. Cambridge: Cambridge Press.

NAS (2010) Rising above the gathering storm revisited: rapidly approaching category 5. Washington: The National Academic Press.

Nelson, R. (1992) "Recent writes on competitiveness: boxing the compass”. California Management Review.

Nelson, R. (1996[2006]) As fontes do crescimento econômico. Campinas: Unicamp

OCDE (1992) Technology and the economy; the key relationships. Paris: OCDE

OCDE (1996) Globalisation and Competitiveness. Paris: OCDE.

OCDE (1999) The Globalization of industry in the OECD countries. Paris: OCDE.

Possas, M. L. (1985) Estruturas de mercado em oligopólio. São Paulo: Hucitec.

Possas, M. S. (1999) Concorrência e competitividade: notas sobre estratégias e dinâmica seletiva na economia capitalista. São Paulo: Hucitec.

Schumpetr, J. A. (1943[1984]) Capitalismo, socialismo e democracia. Rio de Janeiro: Zahar

Silverberg, G. (1988) "Modelling economic dynamics and technical change: mathematical approaches to self-organisation and evolution”. In: Dosi, G. et al. The Technical change and economic theory. London: Pinter Publishers.

Simon, H. A. (1996) The Sciences of the artificial. Cambridge: MIT Press.

Teece, D. J. et al. (1997) "Dynamic capabilities and strategic management”. Strategic Management Journal, vol. $18, \mathrm{n}^{\circ} 7$, os 509-533.

Teece, D. J. (2007) "Explicating dynamic capabilities: the nature and microfundations of (sustainable) enterprise performance”. Strategic Management Journal, 28, ps. 1319-1350.

Tidd, J., Bessant, J. e Pavitt, K. (2008) Gestão da inovação. Porto Alegre: Bookman. 